Review Article

\title{
Do miRNAs Play a Role in Fetal Growth Restriction? A Fresh Look to a Busy Corner
}

\author{
Benito Chiofalo, ${ }^{1}$ Antonio Simone Laganà, ${ }^{1}$ Alberto Vaiarelli, ${ }^{1}$ \\ Valentina Lucia La Rosa, ${ }^{2}$ Diego Rossetti, ${ }^{3}$ Vittorio Palmara, ${ }^{1}$ \\ Gaetano Valenti, ${ }^{4}$ Agnese Maria Chiara Rapisarda, ${ }^{4}$ Roberta Granese, ${ }^{1}$ \\ Fabrizio Sapia, ${ }^{4}$ Onofrio Triolo, ${ }^{1}$ and Salvatore Giovanni Vitale ${ }^{1}$ \\ ${ }^{1}$ Unit of Gynecology and Obstetrics, Department of Human Pathology in Adulthood and Childhood "G. Barresi", \\ University of Messina, Messina, Italy \\ ${ }^{2}$ Unit of Psychodiagnostics and Clinical Psychology, University of Catania, Catania, Italy \\ ${ }^{3}$ Department of Maternal and Child Health, Gavardo Hospital, Brescia, Italy \\ ${ }^{4}$ Department of General Surgery and Medical Surgical Specialties, University of Catania, Catania, Italy \\ Correspondence should be addressed to Antonio Simone Laganà; antlagana@unime.it
}

Received 31 January 2017; Accepted 20 March 2017; Published 29 March 2017

Academic Editor: Erich Cosmi

Copyright (c) 2017 Benito Chiofalo et al. This is an open access article distributed under the Creative Commons Attribution License, which permits unrestricted use, distribution, and reproduction in any medium, provided the original work is properly cited.

\begin{abstract}
Placenta is the crucial organ for embryo and fetus development and plays a critical role in the development of fetal growth restriction (FGR). There are increasing evidences on the role of microRNAs (miRNAs) in a variety of pregnancy-related complications such as preeclampsia and FGR. More than 1880 miRNAs have been reported in humans and most of them are expressed in placenta. In this paper, we aimed to review the current evidence about the topic. According to retrieved data, controversial results about placental expression of miRNAs could be due (at least in part) to the different experimental methods used by different groups. Despite the fact that several authors have demonstrated a relatively easy and feasible detection of some miRNAs in maternal whole peripheral blood, costs of these tests should be reduced in order to increase cohorts and have stronger evidence. In this regard, we take the opportunity to solicit future studies on large cohort and adequate statistical power, in order to identify a panel of biomarkers on maternal peripheral blood for early diagnosis of FGR.
\end{abstract}

\section{Introduction}

Fetal growth restriction (FGR) refers to a condition in which there is the stop or the decrease of the genetic determined potential growth of a fetus during pregnancy. FGR is due to different causes, including maternal smoking, undernutrition, infection, and congenital abnormalities; conversely, if it is not possible to individuate a clear cause, it is defined as idiopathic. FGR is often associated with preeclampsia [1] and represents the most common pregnancy complication, accounting for about a third of all preterm births. Usually FGR is diagnosed when, through ultrasound, fetal weight is estimated to be less than the 10th percentile for gestational age using a validated fetal growth scale [2]. The most studied cause of FGR in animal models is maternal calorie restriction during gestation, but while much is known about the consequences of this deprivation, molecular mechanisms that underlie these conditions still remain unclear.

Placenta is the crucial organ for embryo and fetus development and plays a critical role in the development of FGR. In this regard, FGR could be considered as a placentation disorder, derived from a dysregulation in trophoblast invasion with characteristic tissue morphology that leads to uteroplacental insufficiency. This condition would greatly benefit from the availability of early diagnostic tests to give an opportunity for early intervention or prevention, to improve maternal-fetal outcomes, and to substantially contain the public health costs. 
There are increasing evidences on the role of microRNAs (miRNAs) in a variety of pregnancy-related complications such as preeclampsia and fetal growth restriction. More than 1880 miRNAs have been reported in humans and most of them are expressed in placenta. This kind of nucleic acid belongs to the family of small noncoding RNAs of on average 22 nucleotides in length, which regulates gene expression at the posttranscriptional level, inhibiting translation or promoting specific mRNAs degradation through interaction with the $3^{\prime}$ untranslated region $[3,4]$. In detail, miRNAs seem to modulate cell development, differentiation, and proliferation, cell type-specific function, and are involved in the pathogenesis of many human diseases [5]. In several cases, miRNA expression is tissue-specific and, in addition, is significantly different between physiology and pathological conditions: for these reasons, investigations about miRNAs gained increasing attention for the possibility of future application in clinical diagnostics $[6,7]$.

Starting from these considerations, we aimed to review the current literature focusing on the role of miRNAs in FGR.

\section{Materials and Methods}

We performed a selective literature search of articles in English language, published from 2002 to 2017 and indexed in PubMed. We searched the following Medical Subject Headings (MeSH): "MicroRNAs" AND "Fetal Growth Retardation". The initial database screening was performed by three authors (Laganà AS, Vaiarelli A, La Rosa VL), who were blinded to the aim of the study. Subsequently, other three authors (Chiofalo B, Rossetti D, Vitale SG) selected relevant information from the screened literature. We considered eligible all original manuscripts (randomized, observational, and retrospective studies), case series, and case reports. Furthermore, we extracted relevant information from selected reviews.

\section{Results}

3.1. Placental MicroRNAs. Several studies focused their attention on the expression of different miRNAs in placentas using real-time-PCR. Cindrova-Davies et al. [8] analyzed miRNA21 expression from placentas of a small cohort $(n=6)$ of early-onset FGR cases and found its significant upregulation. Guo et al. [9] identified a significant downregulation of miRNA-194 in placentas from 26 FGR cases and from 16 preeclamptic women (16), compared to those from 29 normal pregnancies (29). Hromadnikova et al. [10] for the first time explored, in two different experiments, the placental expression profile of miRNAs known to be involved in cardiovascular and cerebrovascular diseases. They found that upregulation of miR-499a-5p is a common feature of all placental insufficiencies such as preeclampsia $(n=80)$, gestational hypertension $(n=35)$, and FGR $(n=35)$; in addition, they demonstrated an upregulation of miR-1-3p in FGR pregnancies with abnormal umbilical fetal flows $(n=19)$; finally, they found downregulation of a series of miRNAs (miR16-5p, miR-26a-5p, miR-100-5p, miR-103a-3p, miR-122-5p,
miR-125b-5p, miR-126-3p, miR-143-3p, miR-145-5p, miR-1955p, miR-199a-5p, miR-221-3p, miR-342-3p, and miR-574-3p) in FGR requiring the delivery before 34 weeks of gestation.

Other authors studied miRNA-424 and its target gene (mitogen-activated protein kinase) that play a role in endothelial cell proliferation through fibroblast growth factor receptor 1 and regulate vascular endothelial growth factor [11]. According to their data analysis, the levels of this miRNA are increased in placentas from 25 FGR pregnancies compared with 25 placentas from uncomplicated pregnancies, suggesting that miRNA-424 is involved in placental disorders. Another study by $\mathrm{Su}$ et al. [12] searched, in a cohort of placentas, the miRNAs that regulate endocrine gland derived vascular endothelial growth factor (EG-VEGF) expression: they concluded that miR-346 and miR-582-3p regulate EG-VEGF-induced trophoblast invasion through repressing metalloproteinases 2 and 9. In addition, FGR placental tissues show an aberrant high expression level of miR-141, suggesting that this miRNA might play important roles in the pathogenesis of the disease by suppressing E2F transcription factor 3 and pleomorphic adenoma gene 1 [13].

To date, many studies focused their attention on chromosome 19 miRNA cluster (C19MC) [14-16]. In detail, C19MC comprises 46 miRNAs and is the largest gene cluster of miRNAs in humans, exclusively expressed in undifferentiated cells and in placenta. In this regard, comparing 14 placentas from FGR pregnancies with 14 from normal pregnancies, it was recently found that hypoxic stress does not affect C19MC miRNA expression, except for downregulation of miR-500c$3 p$ [14]. Similarly, Hromadnikova et al. [17] detected a downregulation of 6 miRNAs (miR-517-5p, miR-518f-5p, miR519a, miR-519d, miR-520a-5p, and miR-525) in placental tissues of 36 FGR pregnancies: compared to the previous studies, these results seem more robust since that authors investigated more types of miRNAs and used those that were previously demonstrated to be exclusively expressed or highly expressed in placental tissues. The significantly decreased expression of miR-519d, but not of miR-520a-5p and miR-525, was also confirmed by others on a larger cohort (50 healthy pregnancies compared with 45 FGR cases) [15]. Nevertheless, other experiments found that the expression of miR-518b was decreased, whereas miR-519a was significantly increased, in 30 FGR placentas [16]. Some of these miRNAs studied in human placentae were also studied in animal models.

3.2. Circulating miRNAs. During pregnancy, due to a normal extravillous trophoblast invasion, nucleic acids of the placental compartment are released into the maternal circulation: this release occurs through the migration of microvesicles from apoptotic/necrotic cells and active cellular communication system, involving also nanovesicles/exosomes and subcellular fragments $[18,19]$. Due to placental continuous remodeling, these extracellular nucleic acids may be detected in maternal blood during the course of gestation and can be measured to monitor placental function and allow early diagnosis of pregnancy complications [20-23]. 
For these motivations in recent years there has been a trend to develop noninvasive methods for the detection in maternal circulation of cell-free nucleic acids, including miRNAs coming from the embryo-placental compartment [24-43]. Some studies detected FGR-specific miRNA expression changes in placentas, but these differences were not detectable in plasma [15-44]. A significant elevation of several extracellular placenta-specific miRNA levels was recently showed (miR-516-5p, miR-517, miR-518b, miR-520a, miR-520h, miR-525, and miR-526a,) during early gestation in 7 pregnancies with later onset of preeclampsia and/or FGR [44]. According to these data, an early screening (i.e., within the 12th to 16th weeks) of miRNA circulating levels may differentiate between women with normally progressing pregnancies and those who could later develop placental insufficiency-related complications [44]. Nevertheless, recent data showed that C19MC microRNAs might play a role in the pathogenesis of preeclampsia, but not of FGR [45]. Last year, Hromadnikova's group investigated maternal blood levels of specific miRNAs involved in cardiovascular and cerebrovascular diseases, finding a downregulation of miR-100-5p, miR-125b-5p, and miR-199a-5p in 39 patients with gestational hypertension, in 68 with preeclampsia, and in 33 with fetal growth restriction compared with 55 healthy controls; in addition, they showed downregulation of miR-17-5p, miR-146a-5p, miR-221-3p, and miR574-3p only in FGR pregnancies [46]. In a small-scale analysis, others found that a group of miRNAs that are altered by hypoxia in trophoblasts (miR-27a, miR-30d, miR141, miR-200c, miR-424, miR-205 and miR-451, miR-491, miR-517a, miR-518b, miR-518e, and miR-524) is elevated in FGR pregnancies ( $n=14$ FGR versus $n=14$ controls) [47].

Some of these miRNAs, such as miR-141, miR-200c, and miR-205, were studied also in animal models $[48,49]$. In particular, it was found that they play important roles in the maintenance of the integrity of the folded trophoblastendometrial epithelial bilayer in porcine placentas [48].

\section{Discussion}

Based on the abovementioned data, miRNAs seem to be involved in placental development and consequently in placenta related disorders. As showed in Table 1, controversial results among these studies in placental expression of miRNAs could be due, at least in part, to the different experimental methods used by different groups. Despite the fact that several authors have demonstrated a relatively easy and feasible detection of some miRNAs in maternal whole peripheral blood [44-47], costs of these tests should be reduced in order to increase cohorts and have stronger evidence.

In this regard, we acknowledge that it may be extremely important to address future research directions taking into account the already available data from in vitro experiments and animal models: indeed, accumulating evidence suggests that miR141-3p and miR-200a-3p play a pivotal role for placental development in mouse and regulate the expression of insulin-like growth factor 2 [50]. Interestingly, upregulation of miR-125b was found to reduce significantly ethanol-induced caspase-3 activation and to diminish ethanol-induced growth retardation in mouse embryos [51], suggesting a possible protective role that is worthy of further investigation. Conversely, miR-24 and miR-103-2, which are related to adipocyte development, were both increased in low birth weight male guinea pig pups [52]. Probably this last element could be further confirmed in future studies, since several sex-specific effects were already found to be more pronounced in males with respect to females [53]. Last but not least, recent data showed that FGR is associated with increased lung miR-126$3 p$ levels, which is known to modulate the expression of angiogenic factor, in rats [54]. The importance of angiogenic regulatory pathways was also highlighted by the abnormal upregulated expression of miR-127, miR-21, and miR16 in placentas of deceased cloned sheep with respect to controls [55]. These data are extremely fascinating, since miR-21 expression was associated with increased vascular resistance also in growth-restricted human pregnancies $[8$, 56].

As suggested by accumulating evidence, miRNAs play also a pivotal role in epigenetic processes $[57,58]$. Epigenetic mechanisms include DNA methylation, imprinting, and RNA transcriptional regulation through RNA molecules, such as miRNAs. These processes are influenced by multiple factors: intrauterine nutrient availability (determined by maternal nutrition and placental function) [59-62], maternal age $[63,64]$, use of drugs $[65,66]$, endocrine disruptors [67], toxins, and infectious agents. For this reason, integrated assessment of early pregnancy should evaluate a combination of biomarkers and ultrasound [68-73]. In addition, we take the opportunity to stress how future investigations about miRNA levels in both sera and placentas should evaluate the possible overlapping among preeclampsia, FGR, and gestational diabetes, since they all have in common placental vascular alterations due to angiogenic disbalance [74].

It is however clear that epigenetic information is transmitted, and potentially inherited, across generations through the remodeling of chromatin states. In this regard, selective miRNA expression may be involved in FGR through epigenetic mechanism.

\section{Conclusion}

Understanding which miRNAs are associated with the onset/ progression of FGR seems mandatory to improve early diagnosis and management of the disease. In this regard, we take the opportunity to solicit future studies on large cohort and adequate statistical power, in order to identify a panel of biomarkers on maternal peripheral blood for early diagnosis of FGR.

\section{Conflicts of Interest}

The authors declare that there are no conflicts of interest regarding the publication of this paper. 


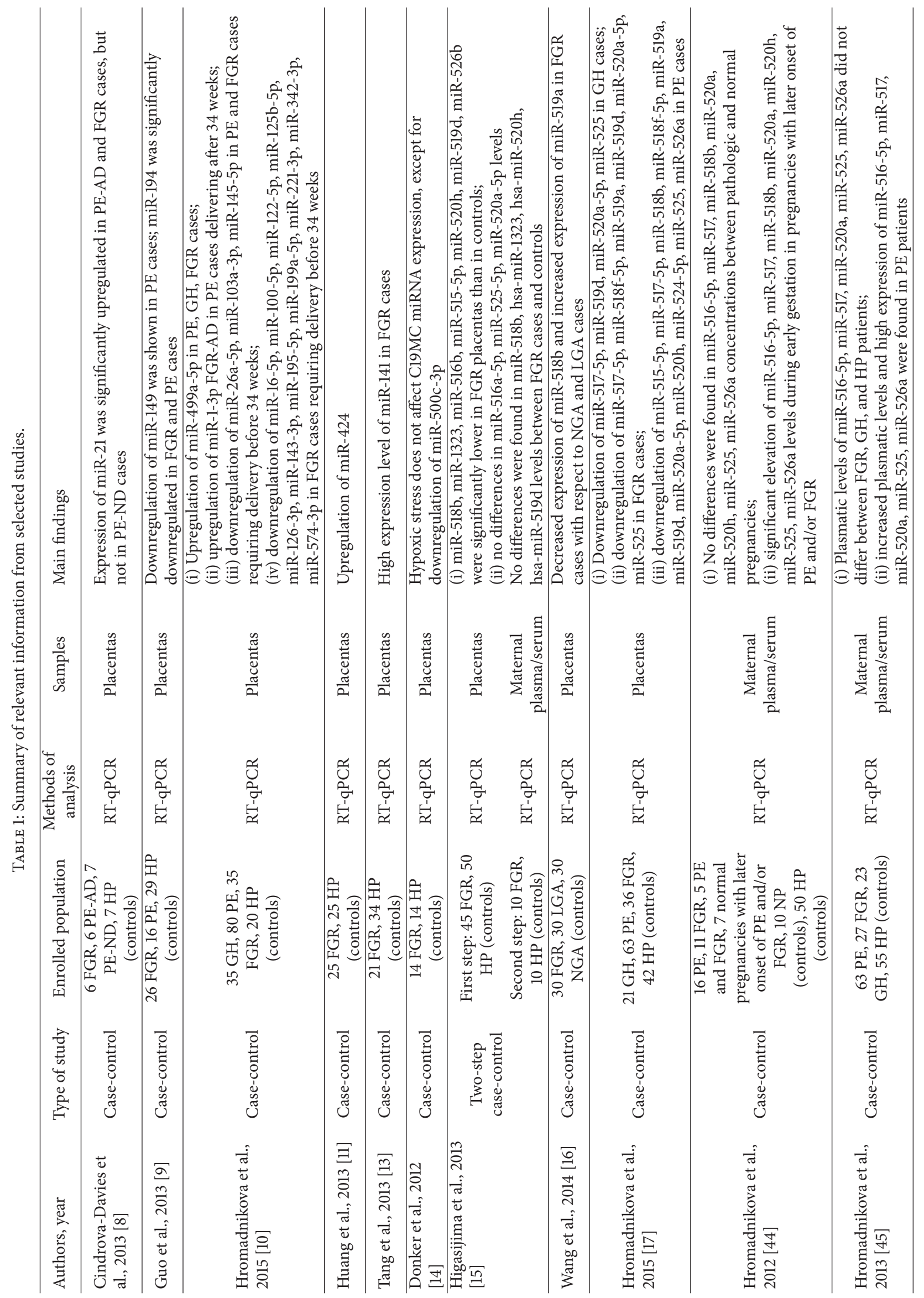




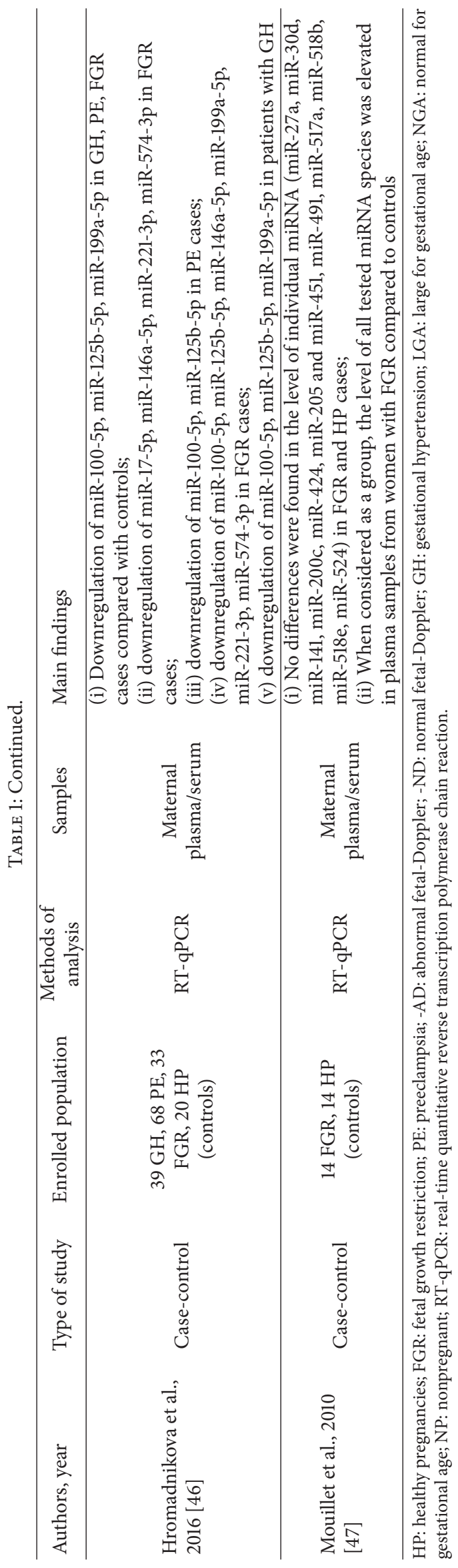




\section{References}

[1] J. Villar, G. Carroli, D. Wojdyla et al., "Preeclampsia, gestational hypertension and intrauterine growth restriction, related or independent conditions?" American Journal of Obstetrics and Gynecology, vol. 194, no. 4, pp. 921-931, 2006.

[2] F. P. Hadlock, R. L. Deter, and R. B. Harrist, "Sonographic detection of abnormal fetal growth patterns," Clinical Obstetrics and Gynecology, vol. 27, no. 2, pp. 342-351, 1984.

[3] E. C. Lai, "Micro RNAs are complementary to 3 ' UTR sequence motifs that mediate negative post-transcriptional regulation," Nature Genetics, vol. 30, no. 4, pp. 363-364, 2002.

[4] D. P. Bartel, "MicroRNAs: genomics, biogenesis, mechanism, and function," Cell, vol. 116, no. 2, pp. 281-297, 2004.

[5] A. Ichihara, M. Jinnin, K. Yamane et al., "microRNA-mediated keratinocyte hyperproliferation in psoriasis vulgaris," British Journal of Dermatology, vol. 165, no. 5, pp. 1003-1010, 2011.

[6] G. A. Calin and C. M. Croce, "MicroRNAs and chromosomal abnormalities in cancer cells," Oncogene, vol. 25 , no. 46, pp. 6202-6210, 2006.

[7] N. Rosenfeld, R. Aharonov, E. Meiri et al., "MicroRNAs accurately identify cancer tissue origin," Nature Biotechnology, vol. 26, no. 4, pp. 462-469, 2008.

[8] T. Cindrova-Davies, E. A. Herrera, Y. Niu, J. Kingdom, D. A. Giussani, and G. J. Burton, "Reduced cystathionine $\gamma$ lyase and increased miR-21 expression are associated with increased vascular resistance in growth-restricted pregnancies: hydrogen sulfide as a placental vasodilator," American Journal of Pathology, vol. 182, no. 4, pp. 1448-1458, 2013.

[9] L. Guo, S. Q. Tsai, N. E. Hardison et al., "Differentially expressed microRNAs and affected biological pathways revealed by modulated modularity clustering (MMC) analysis of human preeclamptic and IUGR placentas," Placenta, vol. 34, no. 7, pp. 599-605, 2013.

[10] I. Hromadnikova, K. Kotlabova, L. Hympanova, and L. Krofta, "Cardiovascular and cerebrovascular disease associated microRNAS are dysregulated in placental tissues affected with gestational hypertension, preeclampsia and intrauterine growth restriction," PLoS ONE, vol. 10, no. 9, Article ID e0138383, 2015.

[11] L. Huang, Z. Shen, Q. Xu, X. Huang, Q. Chen, and D. Li, "Increased levels of microRNA-424 are associated with the pathogenesis of fetal growth restriction," Placenta, vol. 34, no. 7, pp. 624-627, 2013.

[12] M. Su, P. Tsai, H. Tsai, Y. Chen, and P. Kuo, "miR-346 and miR-582-3p-regulated EG-VEGF expression and trophoblast invasion via matrix metalloproteinases 2 and 9," BioFactors, 2016.

[13] Q. Tang, W. Wu, X. Xu et al., "miR-141 contributes to fetal growth restriction by regulating PLAG1 expression," PLoS ONE, vol. 8, no. 3, Article ID e58737, 2013.

[14] R. B. Donker, J. F. Mouillet, T. Chu et al., "The expression profile of C19MC microRNAs in primary human trophoblast cells and exosomes," Molecular Human Reproduction, vol. 18, no. 8, pp. 417-424, 2012.

[15] A. Higashijima, K. Miura, H. Mishima et al., "Characterization of placenta-specific microRNAs in fetal growth restriction pregnancy," Prenatal Diagnosis, vol. 33, no. 3, pp. 214-222, 2013.

[16] D. Wang, Q. Na, W.-W. Song, and G.-Y. Song, "Altered expression of miR-518b and miR-519a in the placenta is associated with low fetal birth weight," American Journal of Perinatology, vol. 31, no. 9, pp. 729-734, 2014.
[17] I. Hromadnikova, K. Kotlabova, M. Ondrackova et al., "Expression profile of C19MC microRNAs in placental tissue in pregnancy-related complications," DNA and Cell Biology, vol. 34, no. 6, pp. 437-457, 2015.

[18] C. W. G. Redman, D. S. Tannetta, R. A. Dragovic et al., "Review: does size matter? placental debris and the pathophysiology of pre-eclampsia," Placenta, vol. 33, supplement, pp. S48-S54, 2012.

[19] Y. Ouyang, J.-F. Mouillet, C. B. Coyne, and Y. Sadovsky, "Review: placenta-specific microRNAs in exosomes-good things come in nano-packages," Placenta, vol. 35, supplement, pp. S69-S73, 2014.

[20] D. M. Nelson, "Apoptotic changes occur in syncytiotrophoblast of human placental villi where fibrin type fibrinoid is deposited at discontinuities in the villous trophoblast," Placenta, vol. 17, no. 7, pp. 387-391, 1996.

[21] B. Huppertz and J. C. P. Kingdom, "Apoptosis in the trophoblast-role of apoptosis in placental morphogenesis," Journal of the Society for Gynecologic Investigation, vol. 11, no. 6, pp. 353-362, 2004.

[22] A. F. Orozco, F. Z. Bischoff, C. Horne, E. Popek, J. L. Simpson, and D. E. Lewis, "Hypoxia-induced membrane-bound apoptotic DNA particles: potential mechanism of fetal DNA in maternal plasma," Annals of the New York Academy of Sciences, vol. 1075, pp. 57-62, 2006.

[23] I. Hromadnikova, "Extracellular nucleic acids in maternal circulation as potential biomarkers for placental insufficiency," DNA and Cell Biology, vol. 31, no. 7, pp. 1221-1232, 2012.

[24] Y. M. Dennis Lo, N. Corbetta, P. F. Chamberlain et al., "Presence of fetal DNA in maternal plasma and serum," The Lancet, vol. 350, no. 9076, pp. 485-487, 1997.

[25] M. Smid, A. Vassallo, F. Lagona et al., "Quantitative analysis of fetal DNA in maternal plasma in pathological conditions associated with placental abnormalities," Annals of the New York Academy of Sciences, vol. 945, pp. 132-137, 2001.

[26] E. Caramelli, N. Rizzo, M. Concu et al., "Cell-free fetal DNA concentration in plasma of patients with abnormal uterine artery Doppler waveform and intrauterine growth restriction-a pilot study," Prenatal Diagnosis, vol. 23, no. 5, pp. 367-371, 2003.

[27] J.-M. Costa, A. Benachi, and E. Gautier, "New strategy for prenatal diagnosis of X-linked disorders," The New England Journal of Medicine, vol. 346, no. 19, p. 1502, 2002.

[28] R. J. P. Rijnders, C. E. van der Schoot, B. Bossers, M. A. M. J. de Vroede, and G. C. M. L. Christiaens, "Fetal sex determination from maternal plasma in pregnancies at risk for congenital adrenal hyperplasia," Obstetrics and Gynecology, vol. 98, no. 3, pp. 374-378, 2001.

[29] B. H. W. Faas, E. A. Beuling, G. C. M. L. Christiaens, A. E. G. K. Von Dem Borne, and C. E. Van Der Schoot, "Detection of fetal RHD-specific sequences in maternal plasma," Lancet, vol. 352, no. 9135, p. 1196, 1998.

[30] Y. M. D. Lo, N. M. Hjelm, C. Fidler et al., "Prenatal diagnosis of fetal RhD status by molecular analysis of maternal plasma," The New England Journal of Medicine, vol. 339, no. 24, pp. 1734-1738, 1998.

[31] I. Hromadnikova, L. Vechetova, K. Vesela, B. Benesova, J. Doucha, and R. Vlk, "Non-invasive fetal RHD and RHCE genotyping using real-time PCR testing of maternal plasma in RhD-negative pregnancies," Journal of Histochemistry and Cytochemistry, vol. 53, no. 3, pp. 301-305, 2005. 
[32] K. C. A. Chan, C. Ding, A. Gerovassili et al., "Hypermethylated RASSF1A in maternal plasma: a universal fetal DNA marker that improves the reliability of noninvasive prenatal diagnosis," Clinical Chemistry, vol. 52, no. 12, pp. 2211-2218, 2006.

[33] Y. M. D. Lo, T. N. Leung, M. S. C. Tein et al., "Quantitative abnormalities of fetal DNA in maternal serum in preeclampsia," Clinical Chemistry, vol. 45, no. 2, pp. 184-188, 1999.

[34] T.-W. Lau, T. N. Leung, L. Y. S. Chan et al., "Fetal DNA clearance from maternal plasma is impaired in preeclampsia," Clinical Chemistry, vol. 48, no. 12, pp. 2141-2146, 2002.

[35] B. M. Byrne, A. Crowley, F. Taulo, J. Anthony, J. J. O'Leary, and C. O'Herlihy, "Fetal DNA quantitation in peripheral blood is not useful as a marker of disease severity in women with preeclampsia," Hypertension in Pregnancy, vol. 22, no. 2, pp. 157164, 2003.

[36] A. Sekizawa, M. Jimbo, H. Saito et al., "Cell-free fetal DNA in the plasma of pregnant women with severe fetal growth restriction," American Journal of Obstetrics and Gynecology, vol. 188, no. 2, pp. 480-484, 2003.

[37] D. W. Y. Tsui, K. C. A. Chan, S. S. C. Chim et al., "Quantitative aberrations of hypermethylated RASSF1A gene sequences in maternal plasma in pre-eclampsia," Prenatal Diagnosis, vol. 27, no. 13, pp. 1212-1218, 2007.

[38] I. Hromadnikova, M. Benesova, L. Zejskova et al., "The effect of DYS-14 copy number variations on extracellular fetal DNA quantification in maternal circulation," DNA and Cell Biology, vol. 28, no. 7, pp. 351-358, 2009.

[39] I. Hromadnikova, L. Zejskova, K. Kotlabova et al., "Quantification of extracellular DNA using hypermethylated RASSF1A, SRY, and GLO sequences-evaluation of diagnostic possibilities for predicting placental insufficiency," DNA and Cell Biology, vol. 29, no. 6, pp. 295-301, 2010.

[40] B. L. Pineles, R. Romero, D. Montenegro et al., "Distinct subsets of microRNAs are expressed differentially in the human placentas of patients with preeclampsia," American Journal of Obstetrics and Gynecology, vol. 196, no. 3, pp. 261.el-261.e6, 2007.

[41] X.-M. Zhu, T. Han, I. L. Sargent, G.-W. Yin, and Y.-Q. Yao, "Differential expression profile of microRNAs in human placentas from preeclamptic pregnancies vs normal pregnancies," American Journal of Obstetrics and Gynecology, vol. 200, no. 6, pp. 661.el-661.e7, 2009.

[42] Y. Hu, P. Li, S. Hao, L. Liu, J. Zhao, and Y. Hou, "Differential expression of microRNAs in the placentae of Chinese patients with severe pre-eclampsia," Clinical Chemistry and Laboratory Medicine, vol. 47, no. 8, pp. 923-929, 2009.

[43] S. S. C. Chim, T. K. F. Shing, E. C. W. Hung et al., "Detection and characterization of placental microRNAs in maternal plasma," Clinical Chemistry, vol. 54, no. 3, pp. 482-490, 2008.

[44] I. Hromadnikova, K. Kotlabova, J. Doucha, K. Dlouha, and L. Krofta, "Absolute and relative quantification of placentaspecific microRNAs in maternal circulation with placental insufficiency-related complications," Journal of Molecular Diagnostics, vol. 14, no. 2, pp. 160-167, 2012.

[45] I. Hromadnikova, K. Kotlabova, M. Ondrackova et al., "Circulating C19MC MicroRNAs in preeclampsia, gestational hypertension, and fetal growth restriction," Mediators of Inflammation, vol. 2013, Article ID 186041, 2013.

[46] I. Hromadnikova, K. Kotlabova, L. Hympanova, and L. Krofta, "Gestational hypertension, preeclampsia and intrauterine growth restriction induce dysregulation of cardiovascular and cerebrovascular disease associated microRNAs in maternal whole peripheral blood," Thrombosis Research, vol. 137, pp. 126140, 2016.

[47] J.-F. Mouillet, T. Chu, C. A. Hubel, D. M. Nelson, W. T. Parks, and Y. Sadovsky, "The levels of hypoxia-regulated microRNAs in plasma of pregnant women with fetal growth restriction," Placenta, vol. 31, no. 9, pp. 781-784, 2010.

[48] R. Liu, M. Wang, L. Su, X. Li, S. Zhao, and M. Yu, "The expression pattern of micrornas and the associated pathways involved in the development of porcine placental folds that contribute to the expansion of the exchange surface area," Biology of Reproduction, vol. 93, no. 3, article 62, 2015.

[49] L. Su, S. Zhao, M. Zhu, and M. Yu, "Differential expression of microRNAs in porcine placentas on Days 30 and 90 of gestation," Reproduction, Fertility and Development, vol. 22, no. 8, pp. 1175-1182, 2010.

[50] S. Saha, J. Choudhury, and R. Ain, "MicroRNA-141-3p and miR200a-3p regulate insulin-like growth factor 2 during mouse placental development," Molecular and Cellular Endocrinology, vol. 414, pp. 186-193, 2015.

[51] X. Chen, J. Liu, W.-K. Feng, X. Wu, and S.-Y. Chen, "MiR$125 \mathrm{~b}$ protects against ethanol-induced apoptosis in neural crest cells and mouse embryos by targeting Bak 1 and PUMA," Experimental Neurology, vol. 271, pp. 104-111, 2015.

[52] O. Sarr, J. A. Thompson, L. Zhao, T.-Y. Lee, and T. R. H. Regnault, "Low birth weight male guinea pig offspring display increased visceral adiposity in early adulthood," PLOS ONE, vol. 9, no. 6, Article ID e98433, 2014.

[53] P.-Y. Chen, A. Ganguly, L. Rubbi et al., "Intrauterine calorie restriction affects placental DNA methylation and gene expression," Physiological Genomics, vol. 45, no. 14, pp. 565-576, 2013.

[54] X. Liu, Y. Lin, B. Tian, J. Miao, C. Xi, and C. Liu, "Maternal protein restriction alters VEGF signaling and decreases pulmonary alveolar in fetal rats," International Journal of Clinical and Experimental Pathology, vol. 7, no. 6, pp. 3101-3111, 2014.

[55] W. Ni, S. You, Y. Cao et al., "Aberrant expression of miR-127, miR-21 and miR-16 in placentas of deceased cloned sheep," Research in Veterinary Science, vol. 105, pp. 200-204, 2016.

[56] C. L. Whitehead, W. T. Teh, S. P. Walker, C. Leung, L. Larmour, and S. Tong, "Circulating microRNAs in maternal blood as potential biomarkers for fetal hypoxia in-utero," PLOS ONE, vol. 8, no. 11, Article ID e78487, 2013.

[57] D. S. Fernandez-Twinn, M. Constância, and S. E. Ozanne, "Intergenerational epigenetic inheritance in models of developmental programming of adult disease," Seminars in Cell and Developmental Biology, vol. 43, pp. 85-95, 2015.

[58] S. Tabano, P. Colapietro, I. Cetin et al., "Epigenetic modulation of the IGF2/H19 imprinted domain in human embryonic and extra-embryonic compartments and its possible role in fetal growth restriction," Epigenetics, vol. 5, no. 4, pp. 313-324, 2010.

[59] G. Rizzo, A. S. Laganà, A. M. Rapisarda et al., "Vitamin B12 among vegetarians: status, assessment and supplementation," Nutrients, vol. 8, no. 12, p. 767, 2016.

[60] F. Colonese, V. L. La Rosa, A. S. Laganà, S. G. Vitale, D. Cortinovis, and P. Bidoli, "Comment on: is there a role for vitamin D in human reproduction?” Hormone Molecular Biology and Clinical Investigation, vol. 25, no. 1, pp. 15-28, 2016.

[61] F. Colonese, A. S. Laganà, E. Colonese et al., "The pleiotropic effects of vitamin D in gynaecological and obstetric diseases: an overview on a hot topic," BioMed Research International, vol. 2015, Article ID 986281, 11 pages, 2015. 
[62] F. Corrado, R. D’Anna, A. S. Laganà, and A. Di Benedetto, "Abnormal glucose tolerance later in life in women affected by glucose intolerance during pregnancy," Journal of Obstetrics and Gynaecology, vol. 34, no. 2, pp. 123-126, 2014.

[63] L. Ciancimino, A. S. Laganà, B. Chiofalo, R. Granese, R. Grasso, and O. Triolo, "Would it be too late? A retrospective case-control analysis to evaluate maternal-fetal outcomes in advanced maternal age," Archives of Gynecology and Obstetrics, vol. 290, no. 6, pp. 1109-1114, 2014.

[64] S. G. Vitale, I. Marilli, A. M. Rapisarda, V. Iapichino, F. Stancanelli, and A. Cianci, "Diagnosis, antenatal surveillance and management of prolonged pregnancy: current perspectives," Minerva Ginecologica, vol. 67, no. 4, pp. 365-373, 2015.

[65] A. S. Laganà, F. Sapia, V. L. La Rosa, and S. G. Vitale, "Comment on "Inositols: from physiology to rational therapy in gynecological clinical practice"," Expert Opinion on Drug Metabolism \& Toxicology, vol. 12, no. 12, article 1527, 2016.

[66] S. G. Vitale, P. Rossetti, F. Corrado et al., "How to achieve highquality oocytes? the key role of myo-inositol and melatonin," International Journal of Endocrinology, vol. 2016, Article ID 4987436, 9 pages, 2016.

[67] V. Sofo, M. Götte, A. S. Laganà et al., "Correlation between dioxin and endometriosis: an epigenetic route to unravel the pathogenesis of the disease," Archives of Gynecology and Obstetrics, vol. 292, no. 5, pp. 973-986, 2015.

[68] S. G. Vitale, A. S. Laganà, A. M. Rapisarda et al., "Role of urocortin in pregnancy: an update and future perspectives," World Journal of Clinical Cases, vol. 4, no. 7, pp. 165-171, 2016.

[69] F. Padula, A. S. Laganà, S. G. Vitale et al., "The introduction of the absolute risk for the detection of fetal aneuploidies in the first-trimester screening," Journal of Maternal-Fetal and Neonatal Medicine, 2016.

[70] F. Padula, A. S. Laganà, S. G. Vitale, and C. Giorlandino, "Comment on 'customized fetal growth charts for parents' characteristics, race, and parity by quantile regression analysis: a cross-sectional multicenter Italian study,' Journal of Ultrasound in Medicine, vol. 35, no. 4, p. 855, 2016.

[71] A. S. Laganà, A. Favilli, O. Triolo, R. Granese, and S. Gerli, "Early serum markers of pre-eclampsia: are we stepping forward?" Journal of Maternal-Fetal and Neonatal Medicine, vol. 29, no. 18, pp. 3019-3023, 2016.

[72] F. Libotte, D. Bizzoco, I. Gabrielli et al., "Pallister-Killian syndrome: cytogenetics and molecular investigations of mosaic tetrasomy $12 \mathrm{p}$ in prenatal chorionic villus and in amniocytes. Strategy of prenatal diagnosis," Taiwanese Journal of Obstetrics and Gynecology, vol. 55, no. 6, pp. 863-866, 2016.

[73] P. Cignini, L. M. Savasta, F. A. Gulino et al., "Predictive value of pregnancy-associated plasma protein-A (PAPP-A) and free beta-hCG on fetal growth restriction: results of a prospective study," Archives of Gynecology and Obstetrics, vol. 293, no. 6, pp. 1227-1233, 2016.

[74] L. M. R. Santa, L. Y. G. Teshima, J. V. F. Forero, and A. O. C. Giraldo, "Angiomirs: potential biomarkers of pregnancy's vascular pathologies," Journal of Pregnancy, vol. 2015, Article ID 320386, 10 pages, 2015. 


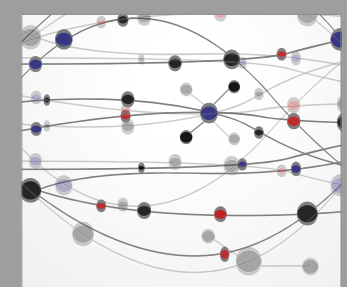

The Scientific World Journal
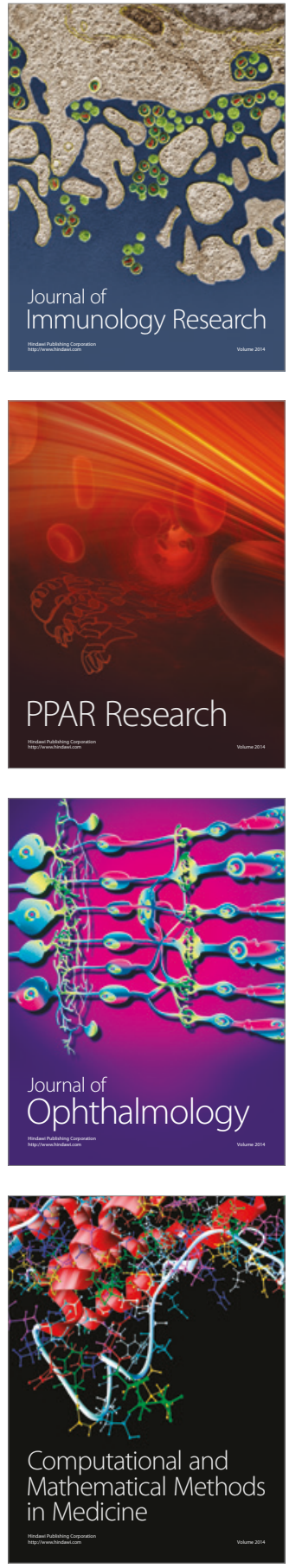

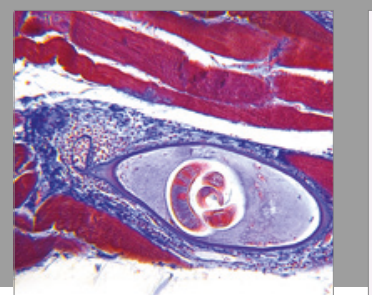

Gastroenterology Research and Practice
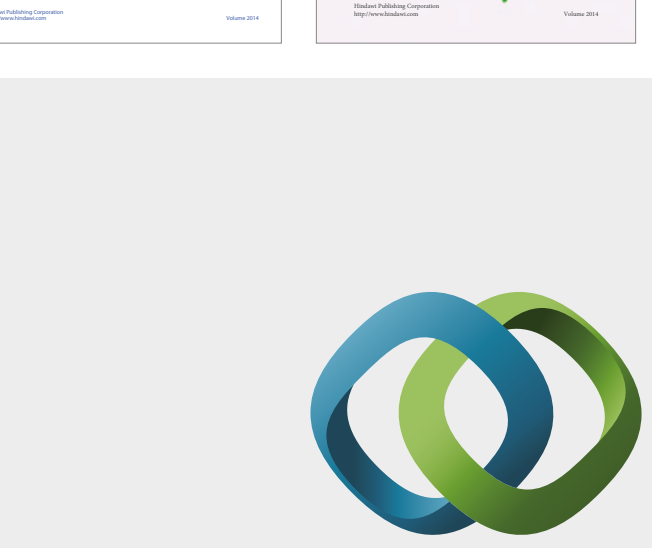

\section{Hindawi}

Submit your manuscripts at

https://www.hindawi.com
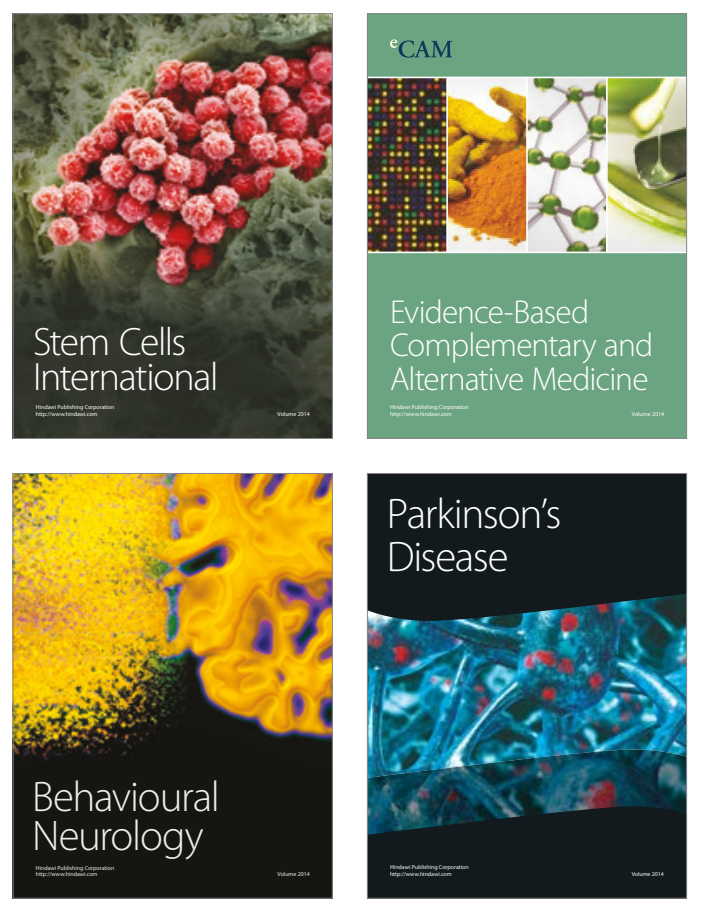
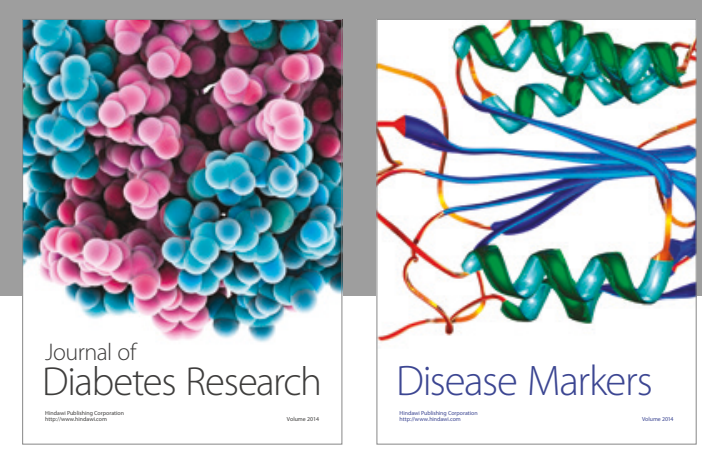

Disease Markers
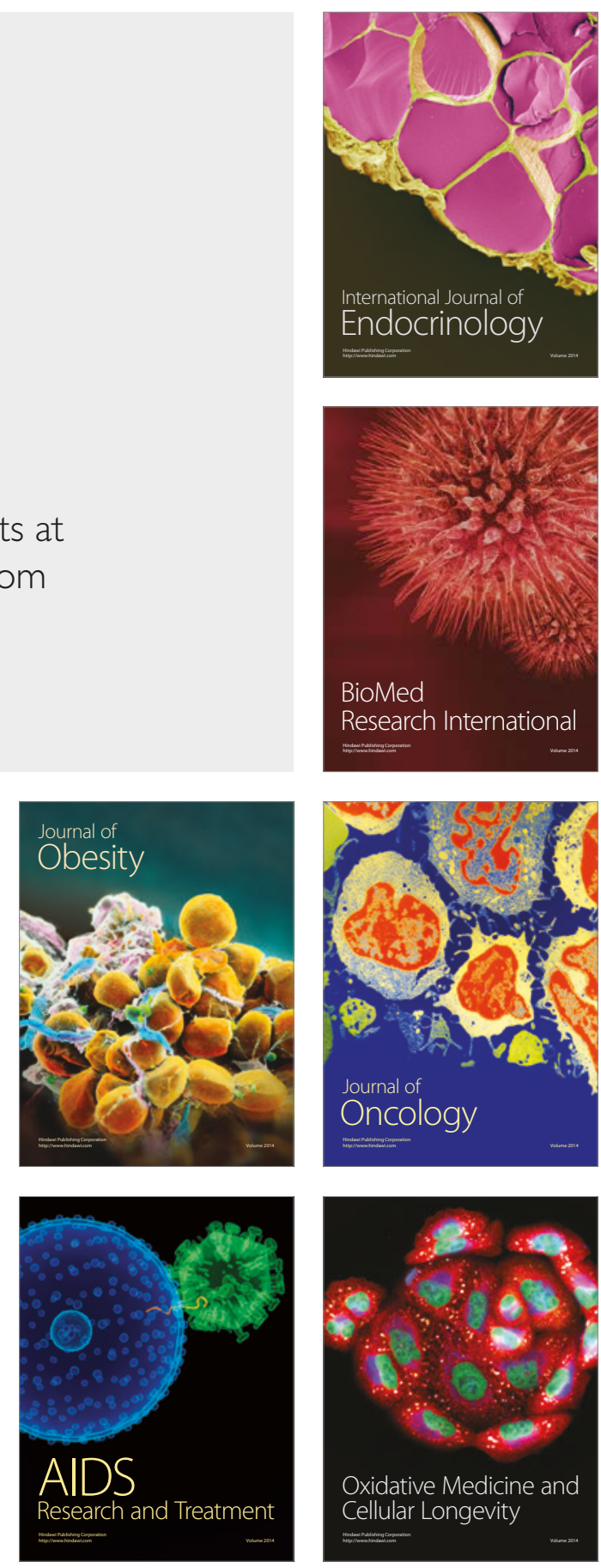